\section{Serum markers and development of delayed neuropsychological sequelae after acute carbon monoxide poisoning: anion gap, lactate, osmolarity, S100B protein, and interleukin-6}

\author{
Hyukhoon Kim ${ }^{1}$, Sangchun Choi ${ }^{1,2}$, Eunjung Park', Eunhui Yoon ${ }^{2}$, \\ Younggi Min ${ }^{1}$, Samsun Lampotang ${ }^{2}$ \\ 'Department of Emergency Medicine, Ajou University School of Medicine, Suwon, Korea \\ ${ }^{2}$ Center for Safety, Simulation \& Advanced Learning Technologies, University of Florida, Gainesville, FL, USA
}

Objective Reliable biomarkers of delayed neuropsychological sequelae (DNS) after acute carbon monoxide (CO) poisoning are lacking. This study investigated the associations between potential serum markers and the development of DNS after acute CO poisoning.

Methods Retrospective chart reviews were conducted for patients diagnosed with acute CO poisoning during a 28-month period. The patients were divided into two groups according to the presence or absence of having developed DNS. Multivariate analysis was performed to identify predictors of DNS after CO poisoning.

Results Of a total of 102 patients, $10(9.8 \%)$ developed DNS. The levels of serum osmolarity, S100B protein, and serum lactate, as well as serum anion gap, were statistically significant in univariate analysis. Multiple logistic regression analysis showed that anion gap (adjusted odds ratio $[A O R], 1.36 ; 95 \%$ confidence interval $[\mathrm{Cl}], 1.11$ to 1.88), serum lactate level (AOR, 1.74; $95 \% \mathrm{Cl}, 1.26$ to 2.75$)$, and serum S100B protein level ([AOR, $7.02 \times 10^{5} ; 95 \% \mathrm{Cl}, 4.56 \times 10^{2}$ to $\left.9.00 \times 10^{10}\right]$ in model $1,\left[A O R, 3.69 \times 10^{5} ; 95 \% \mathrm{Cl}, 2.49 \times 10^{2}\right.$ to $\left.2.71 \times 10^{11}\right]$ in model 2) were independently associated with DNS development.

Conclusion Based on our preliminary results, serum lactate level, serum anion gap, and serum $\mathrm{S} 100 \mathrm{~B}$ protein level in the emergency department could be informative predictors of DNS development in patients with acute $\mathrm{CO}$ poisoning. These markers might have the potential to improve early recognition of DNS in patients with acute $\mathrm{CO}$ poisoning.

Keywords Acid-base equilibrium; Lactic acid; Carbon monoxide poisoning; Neurotoxicity syndromes; Osmolar concentration
eISSN: 2383-4625

Received: 7 March 2017

Revised: 25 August 2017

Accepted: 29 August 2017

Correspondence to: Sangchun Choi Department of Emergency Medicine, Ajou University School of Medicine, 206 World cup-ro, Yeongtong-gu, Suwon 16499, Korea

E-mail: avenue59@ajou.ac.kr

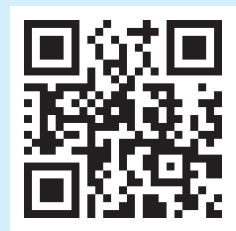

How to cite this article:

Kim H, Choi S, Park E, Yoon E, Min Y, Lampotang $S$. Serum markers and development of delayed neuropsychological sequelae after acute carbon monoxide poisoning: anion gap, lactate, osmolarity, S100B protein, and interleukin-6. Clin Exp Emerg Med 2018;5(3):185-191.

This is an Open Access article distributed under the terms of the Creative Commons Attribution Non-Commercial License (http:// creativecommons.org/licenses/by-nc/4.0/). 


Capsule
Wummary is already known
$\begin{aligned} & \text { Previous studies have demonstrated the prognostic values of interleukin-6, S100B, neuron-specific enolase, and myelin } \\ & \text { basic protein levels as predictors of delayed neuropsychological sequelae (DNS) development. However, these biomarkers } \\ & \text { are available only to limited medical facilities because of the construction costs of high-tech infrastructures. }\end{aligned}$
$\begin{aligned} & \text { What is new in the current study } \\ & \text { Widely available serum anion gap and serum lactate values could be helpful to predict the development of DNS at rela- } \\ & \text { tively early clinical stages. }\end{aligned}$

\section{INTRODUCTION}

Carbon monoxide (CO) poisoning is a significant cause of illness and death. ${ }^{1-4}$ The toxic symptoms and signs of $\mathrm{CO}$ poisoning are highly variable depending on the acuity and the duration of exposure. ${ }^{2}$ Several days to six weeks after a complete or partial recovery from initial hypoxic injuries during the acute stage of $\mathrm{CO}$ poisoning (the "lucid interval"), a sudden occurrence or deterioration of neuropsychological symptoms and signs sometimes develops, which is defined as delayed encephalopathy or delayed neuropsychological sequelae (DNS)., ${ }^{3,4}$ The typical features of DNS include apathy, disorientation, amnesia, mutism, fecal/urinary incontinence, gait disturbance, anxiety, depression, and Parkinsonism. ${ }^{2,5,6}$ DNS development, which is permanent in about $25 \%$ of cases, might lead to poor quality of life in victims. ${ }^{3,5}$ This subsequently increases the socioeconomic burden of individuals and nations. Thus, preventing DNS after acute CO poisoning is of great importance. Previous studies demonstrated that biomarkers such as neuron-specific enolase (NSE), S100B protein, and myelin basic protein (MBP) could be useful in predicting the occurrence of DNS. ${ }^{7-10}$ Although several studies reported a correlation between the cerebrospinal fluid (CSF) levels of interleukin-6 (IL-6) and S100B protein and DNS development, their values were outweighed by the unknown mechanisms of acute $\mathrm{CO}$ poisoning. ${ }^{6,10-13}$ Additionally, high-end or specified equipment and facilities were required to measure the levels of NSE, S100B, MBP, and IL-6 in the CSF or serum. Therefore, additional practical markers to predict the occurrence of DNS in clinical practice are needed.

Acid-base disorders (ABDs) are often indicators for severe systemic disorders including acute kidney injury, major traumas, unstable postoperative conditions, and acute drug intoxications. ${ }^{14-17}$ Serum anion gap (AG) and serum osmolar gap are not only highly sensitive diagnostic tools for distinguishing various $A B D$ s but are also a severity index for ABDs. ${ }^{18}$ Similarly, high levels of serum lactate are associated with morbidity and mortality in diverse populations of critically ill patients and disease entities, including acute CO poisoning. ${ }^{19-24}$ The acid-base balance is influenced by changes in serum lactate levels. Severe acute $\mathrm{CO}$ poisoning could result in failure associated with acid-base imbalance. Thus, acid-base imbalances are likely to reflect changes in the serum AG, lactate, and osmolarity levels in patients with acute $\mathrm{CO}$ poisoning. Therefore, these changes could serve as predictors of DNS development if they reflect the severity of acute $\mathrm{CO}$ poisoning.

The purpose of this study was to investigate associations between the levels of the potential serum markers, AG, lactate, osmolarity, S100B protein, and IL-6 and the development of DNS after acute $\mathrm{CO}$ poisoning.

\section{METHODS}

This retrospective study evaluated all patients who visited the emergency department (ED) of Ajou University Hospital, with approximately 90,000 annual visits, between March 2013 and June 2015. Our institutional review board approved this study (AIRBMED-MDB-16-066) and exempted patient informed consent.

The inclusion criteria were patients above 18 years of age with a final diagnosis of acute $\mathrm{CO}$ poisoning and measurements of serum lactate and serum osmolarity levels at the time of ED admission. The diagnosis of acute $\mathrm{CO}$ poisoning was established based on patient history, physical examination, and blood carboxyhemoglobin levels above $3 \%$ or $10 \%$ in nonsmokers or smokers, respectively. The exclusion criteria were concomitant with high-energy trauma, cancer, inhalation burn, history of myocardial dysfunction, inflammatory diseases and illness known to influence serum osmolarity, and history of alcohol consumption within the preceding 24 hours or serum evidence of alcohol ingestion. 
We divided the patients into two groups with and without DNS. No previous study has proposed specific diagnostic criteria or a scoring system to define DNS. Therefore, we defined DNS as patients showing at least one of the following: delayed onset of Parkinsonism, psychosis, apathy, cognitive dysfunction, memory impairment, difficulty in communication, difficulty in walking, or fecal or urinary incontinence. ${ }^{2-6}$

Standardized data abstraction for medical records was performed by two trained emergency physicians. Discrepancies in the two sets of extracted data, if any, were resolved by a thirdparty physician. The physicians were blinded to the patient's laboratory results. The source of $\mathrm{CO}$, cause of exposure, mental status, Glasgow coma scale at the time of ED admission, symptoms and signs at the time of the ED presentation, suspected myocardial injury, neurologic findings, radiologic findings, and application of targeted temperature management were investigated. The results of laboratory studies including serum blood urea nitrogen (BUN)/creatine, serum electrolytes, serum cardiac enzymes (creatine kinase, creatine kinase-muscle and brain, troponin-I), serum osmolarity, and serum IL-6 levels at the time of the ED admission were also evaluated. Suspected myocardial injuries manifested as elevated cardiac enzyme levels and abnormal electrocardiography findings. The serum osmolarity was measured using a micro-osmometer (Advanced instruments 2020; Advanced Instruments, Norwood, MA, USA). Serum IL-6 levels were measured using a quantitative immunochemistry analyzer (Modular analytics E combinations; Roche Diagnostics $\mathrm{GmbH}$, Manheim, Germany). Serum $\mathrm{S} 100 \mathrm{~B}$ protein levels were measured using a quantitative immunoassay analyzer (Modular analytics E170; Roche Diagnostics, Indianapolis, IN, USA). Serum AG values were calculated using the formula $\left(\left[\mathrm{NA}^{+}\right]+\left[\mathrm{K}^{+}\right]\right)-\left([\mathrm{Cl}]+\left[\mathrm{HCO}^{-}\right]\right)$with each result extracted from serum electrolyte analysis. The serum osmolarity was calculated using the formula $\left(2 \times\left[\mathrm{NA}^{+}\right]+[\right.$glucose/18]+[BUN/2.8]). The osmolar gap was the difference between the measured and the calculated osmolarity. The official readout data from the radiologist were reviewed to identify the presence or absence of abnormal lesions in T2-weighted imaging, fluid-attenuated inversion recovery, and diffusion-weighted imaging in magnetic resonance imaging.

DNS development was identified by investigating for the presence of any DNS symptoms and signs by reviewing medical records or by checking the responses to a DNS questionnaire. The DNS symptoms and signs included Parkinsonism, psychosis, apathy, cognitive dysfunction, memory impairment, difficulty in communication, difficulty in walking, or fecal or urinary incontinence..$^{2-6}$ All questions in the DNS questionnaire were prepared beforehand and were simple yes/no questions. DNS frequently develops after hospital discharge or recurs after initial recovery. Therefore, although the medical record may not show any symptoms and signs of DNS, senior physicians (above PGY3 level) in training in the ED, who did not participate in this study, followed up all patients who had been diagnosed with acute $\mathrm{CO}$ poisoning during the study period. They made phone calls to all of the included patients to determine if the patients had experienced any symptoms or signs of DNS.

We expressed continuous data as means \pm standard deviation or medians (interquartile range, IQR) as appropriate, and categorical data as numbers (\%). Mann-Whitney U-tests and Fisher exact tests were used for continuous and categorical variables, respectively, to assess the significance of intergroup differences. Factors selected from the univariate analysis $(P<0.001)$ were considered potential predictors of the development of DNS. These were further analyzed using single models (simple regression) for the predictions of the mixed model. Receiver operating characteristic curves were constructed to establish the cut-off points of variables for optimal sensitivity and specificity in predicting DNS development. Using the factors that showed correlation in each single model, multiple logistic regression analysis was performed to identify the factors that were potential independent predictors by forward stepwise methods with odds ratio tests. We constructed receiver operating characteristic curves to evaluate the multivariate model that was designed in our study to predict the DNS prevalence. The statistical analysis was performed using the R program software for Mac ver. 3.2.2 (R Foundation, https:// www.r-project.org/).

\section{RESULTS}

\section{General patient characteristics}

A total of 299 patients diagnosed with acute CO poisoning were identified during the study period. Of these, 102 patients were included in the final analysis. The remaining 197 patients were excluded because 1) serum IL-6 levels and serum osmolarity were not measured in 133 patients, 2) seven patients had cardiac arrest, and 3) 57 patients showed serum evidence of alcohol consumption. Among the 102 patients who were included in this study, 82 were admitted to the toxicology division of the ED. The general characteristics of these patients are shown in Table 1. The mean age was $39.71 \pm 16.62$ years, and 62 patients $(60.8 \%)$ were male. There was a statistically significant difference between the groups in age $(P<0.001)$. However, there was no statistically significant difference between both groups regarding sex $(P>0.05)$. The common sources of $\mathrm{CO}$ were fire (46.1\%) and charcoal ignition (36.3\%). The median (IQR) carboxyhemoglobin levels were $23.7 \%$ 
Table 1. Patient characteristics

\begin{tabular}{|c|c|c|c|c|}
\hline Variable & Total $(n=102)$ & DNS $(n=10)$ & Non-DNS $(n=92)$ & P-value \\
\hline Age (yr) & $39.7 \pm 16.6$ & $46.2 \pm 19.6$ & $39.0 \pm 16.2$ & $<0.001$ \\
\hline Sex, male & $62(60.8)$ & $6(60)$ & $56(54.9)$ & 1 \\
\hline Briquet & $11(10.8)$ & $2(2.0)$ & $9(8.8)$ & \\
\hline Ignited charcoal & $37(36.3)$ & $7(6.9)$ & $30(29.4)$ & \\
\hline Fire & $47(46.1)$ & $0(0)$ & $47(46.1)$ & \\
\hline Cause of exposure & & & & 0.090 \\
\hline Accidental & $59(57.8)$ & $3(2.9)$ & $56(54.9)$ & \\
\hline Intentional & $43(42.2)$ & $7(6.9)$ & $36(35.3)$ & \\
\hline Glasgow coma scale & $13.6 \pm 2.9$ & $9.2 \pm 4.0$ & $14.1 \pm 2.3$ & $<0.001$ \\
\hline Myocardial injury & $30(29.4 \%)$ & $9(90 \%)$ & $21(22.8 \%)$ & $<0.001$ \\
\hline Anion gap & $17.6 \pm 4.2$ & $24.3 \pm 7.0$ & $16.9 \pm 3.1$ & $<0.001$ \\
\hline Interleukin-6 (pg/mL) & $5.2(2.3-20.3)$ & $57.0(35.8-196.0)$ & $4.4(2.2-12.2)$ & $<0.001$ \\
\hline Serum lactate (mmol/L) & $2.7 \pm 2.6$ & $7.5 \pm 4.7$ & $2.2 \pm 1.5$ & $<0.001$ \\
\hline $\mathrm{S} 100 \mathrm{~B}(\mu \mathrm{g} / \mathrm{L})$ & $0.12(0.051-0.095)$ & $0.280(0.188-0.686)$ & $0.068(0.049-0.087)$ & $<0.001$ \\
\hline Hyperbaric oxygen therapy & $3 / 102(2.9)$ & 0 & 3/92 (3.3) & 0.620 \\
\hline Targeted temperature managemen & $9(8.8)$ & $8(80)$ & $1(1.1)$ & $<0.001$ \\
\hline
\end{tabular}

Values are presented as mean \pm standard deviation, number (\%), or median (interquartile range).

DNS, delayed neuropsychological sequelae; MRI, magnetic resonance imaging.

(IQR, 12.6 to 45.8$)$ and $3.6 \%(I Q R, 2.1$ to 18.1$)$ for both groups, respectively, a statistically significant difference. The mean $A G$ value was $17.65 \pm 4.20$, and the median IL- 6 concentration was $5.25 \mathrm{pg} / \mathrm{mL}$ (IQR, 2.30 to 20.33) (Table 1). Ninety-nine patients (97.1\%) received $100 \%$ oxygen as an initial treatment in the ED, while only three patients (2.9\%) in the non-DNS group required hyperbaric oxygen therapy (Table 1).

\section{Characteristics of the DNS group}

Ten patients (9.8\%) comprised the DNS group. Compared with those in the non-DNS group, patients in the DNS group had significantly worse mental status and lower Glasgow coma scale scores (Table 1). However, no specific symptoms and signs distinguished between the DNS and non-DNS groups. The rate of suspected myocardial injury, AG value, serum osmolarity level, serum lactate level, serum IL-6 level, and S100B protein level were higher in the DNS group than in the non-DNS group (Table 2). These differences were statistically significant $(P<0.001)$. The rate of abnormal findings on magnetic resonance imaging was significantly higher in the non-DNS group than in the DNS group (57.5\% vs. $42.3 \%, \mathrm{P}<0.001)$.
Table 2. Univariate logistic regression analysis of serum anion gap, serum lactate, serum osmolarity, serum S100B protein, and serum interleukin-6 levels

\begin{tabular}{lcr}
\hline \multirow{2}{*}{ Parameter } & \multicolumn{2}{c}{ Results of each simple model } \\
\cline { 2 - 3 } & Odds ratio $(95 \% \mathrm{Cl})$ & P-value \\
\hline Serum anion gap & $1.04(1.03-1.05)$ & $<0.001$ \\
Serum lactate & $1.50(1.26-1.82)$ & $<0.001$ \\
Serum osmolarity & $1.50(1.26-1.82)$ & 0.017 \\
Serum S100B protein & $3.21(2.50-4.12)$ & $<0.001$ \\
Serum interleukin-6 & $1.00(1.00-1.00)$ & $<0.001$
\end{tabular}

$\mathrm{Cl}$, confidence interval.

\section{Predictive factors of DNS}

Serum AG value, serum lactate level, serum osmolarity, serum S100B protein and serum IL-6 levels were independently associated with DNS development in single logistic regression analysis (Table 2). The cut-off value of each test to predict DNS development is described in Table 3. There was a linear correlation between serum AG and lactate levels; thus, we constructed two final mixed models separating these two factors. Two mixed-logistic regression models (model 1 and 2) were also constructed using a forward stepwise selection technique (Fig. 1). The covariates in- 
Table 3. Cut-off values, sensitivity, and specificity of serum anion gap, serum lactate, serum osmolarity, serum S100B protein, and serum interleukin-6 levels for the prediction of delayed neuropsychological sequelae development

\begin{tabular}{lcccc}
\hline Parameter & Cut-off values & AUC (95\% Cl) & Sensitivity (\%) & Specificity (\%) \\
\hline Serum anion gap & 19.3 & $0.81(0.69-0.92)$ & 90.0 & 84.8 \\
Serum lactate $(\mathrm{mmol} / \mathrm{L})$ & 6.5 & $0.75(0.57-0.93)$ & 70.0 & 97.8 \\
Serum osmolarity $($ Osm/L) & 305 & $0.79(0.65-0.92)$ & 60.0 & 88.0 \\
Serum S100B protein $(\mu \mathrm{g} / \mathrm{L})$ & 0.103 & $0.85(0.73-0.96)$ & 90.0 & 84.8 \\
Serum interleukin-6 $(\mathrm{pg} / \mathrm{mL})$ & 15.8 & $0.92(0.88-0.93)$ & 100.0 & 80.4
\end{tabular}

AUC, area under the curve; $\mathrm{Cl}$, confidence interval.
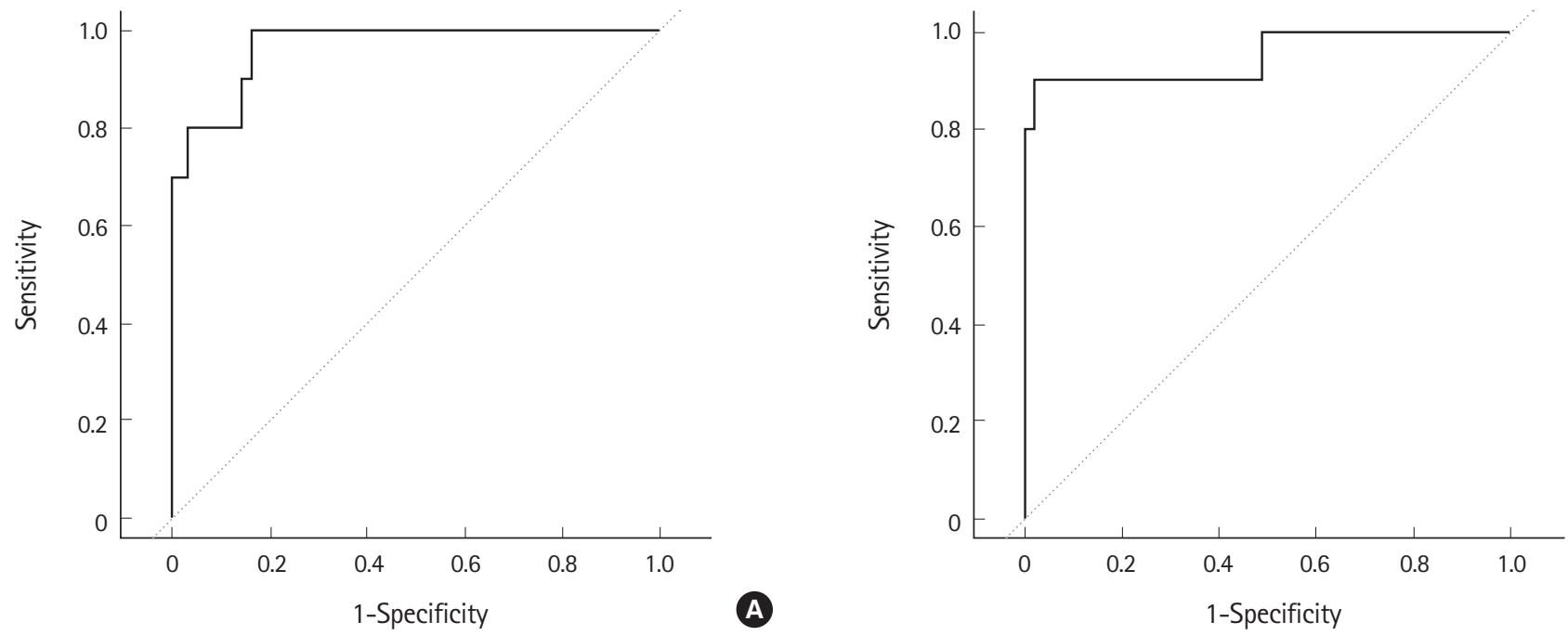

B

Fig. 1. Receiver operating characteristic curves of mixed logistic regression models for the prediction of delayed neuropsychological sequelae development in patients with acute carbon monoxide poisoning. Both Model 1 (area under the curve: 0.9663) and Model 2 (area under the curve: 0.9489) show high discrimination ability with regard to the prediction of delayed neuropsychological sequelae development. Dotted line indicates reference line (A: model 1, B: model 2).

Table 4. Mixed logistic regression analysis with delayed neuropsychological sequelae as a dependent variable in each model

\begin{tabular}{|c|c|c|c|c|}
\hline \multirow{2}{*}{ Parameter } & \multicolumn{2}{|l|}{ Model 1} & \multicolumn{2}{|l|}{ Model 2} \\
\hline & Odds ratio $(95 \% \mathrm{Cl})$ & P-vlaue & Odds ratio $(95 \% \mathrm{Cl})$ & P-vlaue \\
\hline Serum anion gap & $1.36(1.11-1.88)$ & 0.016 & - & - \\
\hline Serum lactate & - & - & $1.74(1.26-2.75)$ & 0.003 \\
\hline Serum S100B protein & $7.02 \times 10^{5}\left(4.56 \times 10^{2}-9.00 \times 10^{10}\right)$ & 0.004 & $3.69 \times 10^{5}\left(2.49 \times 10^{2}-2.71 \times 10^{11}\right)$ & 0.016 \\
\hline
\end{tabular}

$\mathrm{Cl}$, confidence interval.

cluded in these models were serum AG value, serum lactate, and S100B protein levels (Table 4).

\section{DISCUSSION}

In this study, the levels of serum AG, serum lactate, and serum $\mathrm{S} 100 \mathrm{~B}$ protein could predict DNS development after acute CO poisoning. We also proposed cut-off values to predict DNS development after acute $\mathrm{CO}$ poisoning. To the best of our knowledge, this is the first study to evaluate the association between serum $A G$ value and serum osmolarity with predictors for DNS development after acute CO poisoning, although the level of serum osmolarity was not a predictor for DNS development in this study.

Previous studies have demonstrated the prognostic values of IL-6, S100B, NSE, and MBP levels as predictors of DNS development. ${ }^{7-11,23-26}$ However, these biomarkers tests are available only to limited medical facilities because of their costly high-tech infrastructures. Therefore, in this study, we tried to identify practical biomarkers that have both cost-effectiveness and high accessibility in general medical facilities. The value of serum $A G$ and 
serum lactate levels were in accord with this practical purpose. In practical terms, we believe that our findings could be helpful to distinguish patients with DNS from patients at risk of DNS development at relatively early clinical stages.

Serum osmolarity is defined as the number of moles of a substance dissolved in the serum. ${ }^{15,16}$ Previous studies reported increased levels of specific plasma proteins such as MBP, S100B protein, cancer antigen 19-9, intercellular adhesion molecule 1, and macrophage inhibitory protein-1B in patients with DNS after CO poisoning. ${ }^{7,12,27}$ Based on these reports, we assumed that the increases in the levels of variable plasma proteins resulted in increased serum osmolarity in patients with severe acute $\mathrm{CO}$ poisoning; thus, serum osmolarity could be a useful tool for predicting DNS development. We evaluated the applicability of serum osmolarity in this study. However, in multiple logistic regression analysis, no correlation was found between serum osmolarity and DNS development, although there was a statistically significant correlation in univariate analysis (Table 2). Several considerations are needed to interpret this result. First, we could not conduct any quantitative comparisons between serum osmolarity and the rate of DNS development because of the relatively small number of patients with DNS. Second, there was a possibility of co-ingestion with other drugs or substances that could affect the serum osmolarity. It was sometimes impossible to differentiate current illness from suicide attempts by drug overdose due to decreased patient mental status or noncooperation with physician interviews. Therefore, it cannot be concluded whether serum osmolarity was not useful for predicting DNS development in patients with acute CO poisoning.

Although the mechanisms of DNS development remain unclear, one possible mechanism is immune-mediated inflammation induced by IL- 6 during the early stage of acute $\mathrm{CO}$ poisoning, resulting in invasive injuries in the CNS. ${ }^{11,13}$ According to Ide and Kamijo, ${ }^{11}$ IL-6, one of the cytokines regulating immune reactions in the CNS and mainly secreted by microglia and astrocytes, could be a predictive marker for DNS development. Blood-brain barrier disruption by hypoxia and ischemia subject to acute CO poisoning could allow the leakage of a relatively high amount of IL-6 in the CNS to the systemic circulation. ${ }^{28}$ In other words, the degree of brain injury by acute $\mathrm{CO}$ poisoning might be reflected by the serum levels of IL-6. Spinal taps to obtain CSF samples in patients with acute $\mathrm{CO}$ poisoning is somewhat challenging for most emergency physicians. Therefore, we hypothesized that serum levels of IL-6 in patients with DNS might be higher than those in patients without DNS. In Table 2, the median serum IL-6 level in the DNS group was $56.95 \mathrm{pg} / \mathrm{mL}(I Q R, 160.22)$, which was significantly higher than that in the non-DNS group (4.45 pg/mL; IOR, 10.02)
$(P<0.001)$. This difference could be an interesting finding. However, in multiple logistic regression analysis, no correlation was found between the serum levels of IL-6 and DNS development. To our knowledge, this is the first study to assess whether serum levels of IL-6 predict DNS development. However, the results of the present study may have been influenced by the following factors. The time of serum IL-6 measurement was not constant in all patients. We sampled serum IL- 6 based on the time of ED admission. According to Ide and Kamijo, ${ }^{11}$ the CSF levels of IL-6 abruptly increase and swiftly return to normal levels. A one-time sampling of serum IL-6 levels at the time of ED admission might not identify elevated levels after CO injury.

This study has several limitations. First, the results are preliminary because the incidence rate of DNS development was $9.8 \%$ $(10 / 102)$ and the total number of patients with DNS development was relatively small for the multivariate logistic regression analysis. Patients and caregivers sometimes did not recognize cognitive and psychological deficits because the clinical features of DNS vary from mild concentration deficits to insomnia and the definition of DNS development was relatively vague. Therefore, it was possible that a number of patients with late-onset DNS were not included in this study, although we followed up with all patients. Second, the final number of included patients was much smaller than we initially expected. Therefore, our results should be interpreted with caution. This is a complex consequence of the unconfirmed usefulness of biomarkers such as $A G$, osmolarity, and IL-6 for predicting DNS after acute CO poisoning and the nature of retrospective studies. This might have caused a selection bias in our results. In addition, serum AG values and serum lactate levels lacked specificity because they may also be elevated in other diseases. However, false positive findings were not likely to influence the results. Because most of the patients were relatively young and had attempted suicide, no patients were critically ill before they were admitted to our ED. Third, as previously mentioned, co-ingestion of unidentified drugs or substances might influence our results. The toxic alcohol family (e.g., alcohol, methanol, ethylene glycol, and isopropyl alcohol) are the most common substances that influence serum osmolarity. ${ }^{16,18}$ Co-ingestion of these substances might increase serum osmolarity and act as a confounder. We could check the serum alcohol level in our ED. However, this possibility was limited because there was no patient whose clinical course suggested poisoning by these substances or drugs. Fourth, we could not check the coefficients of variations of all laboratory tests before this study. Therefore, there is the possibility of bias or skewness in our results. However, since the quality control of the laboratory tests performed by the laboratory of our hospital undergoes mandatory assessments at regular intervals, 
this was not likely to greatly influence our results.

In conclusion, based on our preliminary results, the serum $A G$, serum lactate, and serum S100B protein levels may be informative predictors of DNS development in patients with acute CO poisoning. These markers could potentially improve early recognition of DNS in patients with acute CO poisoning.

\section{CONFLICT OF INTEREST}

No potential conflict of interest relevant to this article was reported.

\section{REFERENCES}

1. Dolan MC. Carbon monoxide poisoning. CMAJ 1985;133:392-9.

2. Weaver LK. Clinical practice: carbon monoxide poisoning. $\mathrm{N}$ Engl J Med 2009;360:1217-25.

3. Hu H, Pan X, Wan Y, Zhang O, Liang W. Factors affecting the prognosis of patients with delayed encephalopathy after acute carbon monoxide poisoning. Am J Emerg Med 2011;29:261-4.

4. Oh BJ, Im YG, Park E, Min YG, Choi SC. Treatment of acute carbon monoxide poisoning with induced hypothermia. Clin Exp Emerg Med 2016;3:100-4.

5. Jasper BW, Hopkins RO, Duker HV, Weaver LK. Affective outcome following carbon monoxide poisoning: a prospective longitudinal study. Cogn Behav Neurol 2005;18:127-34.

6. Oh S, Choi SC. Acute carbon monoxide poisoning and delayed neurological sequelae: a potential neuroprotection bundle therapy. Neural Regen Res 2015;10:36-8.

7. Ide T, Kamijo Y. Myelin basic protein in cerebrospinal fluid: a predictive marker of delayed encephalopathy from carbon monoxide poisoning. Am J Emerg Med 2008;26:908-12.

8. Ide T, Kamijo Y, Ide A, et al. Elevated S100B level in cerebrospinal fluid could predict poor outcome of carbon monoxide poisoning. Am J Emerg Med 2012;30:222-5.

9. Park E, Ahn J, Min YG, et al. The usefulness of the serum s100b protein for predicting delayed neurological sequelae in acute carbon monoxide poisoning. Clin Toxicol (Phila) 2012;50:183-8.

10. Yardan T, Cevik Y, Donderici O, et al. Elevated serum S100B protein and neuron-specific enolase levels in carbon monoxide poisoning. Am J Emerg Med 2009;27:838-42.

11. Ide T, Kamijo Y. The early elevation of interleukin 6 concentration in cerebrospinal fluid and delayed encephalopathy of carbon monoxide poisoning. Am J Emerg Med 2009;27:992-6.

12. Kamijo Y, Soma K, Ide T. Recurrent myelin basic protein elevation in cerebrospinal fluid as a predictive marker of delayed encephalopathy after carbon monoxide poisoning. Am J Emerg
Med 2007;25:483-5.

13. Park EJ, Min YG, Kim GW, Cho JP, Maeng WJ, Choi SC. Pathophysiology of brain injuries in acute carbon monoxide poisoning: a novel hypothesis. Med Hypotheses 2014;83:186-9.

14. Kitterer D, Schwab M, Alscher MD, Braun N, Latus J. Drug-induced acid-base disorders. Pediatr Nephrol 2015;30:1407-23.

15. Inaba $H$, Hirasawa $H$, Mizuguchi T. Serum osmolality gap in postoperative patients in intensive care. Lancet 1987;1:1331-5.

16. Schelling JR, Howard RL, Winter SD, Linas SL. Increased osmolal gap in alcoholic ketoacidosis and lactic acidosis. Ann Intern Med 1990;113:580-2.

17. Sklar AH, Linas SL. The osmolal gap in renal failure. Ann Intern Med 1983;98:481-2.

18. Kraut JA, Madias NE. Serum anion gap: its uses and limitations in clinical medicine. Clin J Am Soc Nephrol 2007;2:16274.

19. Dezman ZD, Comer AC, Smith GS, Narayan M, Scalea TM, Hirshon JM. Failure to clear elevated lactate predicts 24-hour mortality in trauma patients. J Trauma Acute Care Surg 2015; 79:580-5.

20. Freitas $A D$, Franzon 0. Lactate as predictor of mortality in polytrauma. Arq Bras Cir Dig 2015;28:163-6.

21. Haas SA, Lange T, Saugel B, et al. Severe hyperlactatemia, lactate clearance and mortality in unselected critically ill patients. Intensive Care Med 2016;42:202-10.

22. Jasso-Contreras G, Gonzalez-Velazquez F, Bello-Aguilar L, et al. Lactate levels as a predictor of mortality in patients with septic shock. Rev Med Inst Mex Seguro Soc 2015;53:316-21.

23. Moon JM, Shin MH, Chun BJ. The value of initial lactate in patients with carbon monoxide intoxication: in the emergency department. Hum Exp Toxicol 2011;30:836-43.

24. Cervellin G, Comelli I, Rastelli G, Picanza A, Lippi G. Initial blood lactate correlates with carboxyhemoglobin and clinical severity in carbon monoxide poisoned patients. Clin Biochem 2014; 47:298-301.

25. Brvar M, Mozina $H$, Osredkar J, et al. S100B protein in carbon monoxide poisoning: a pilot study. Resuscitation 2004;61:35760.

26. Rasmussen LS, Poulsen MG, Christiansen M, Jansen EC. Biochemical markers for brain damage after carbon monoxide poisoning. Acta Anaesthesiol Scand 2004;48:469-73.

27. Thom SR, Bhopale VM, Milovanova TM, et al. Plasma biomarkers in carbon monoxide poisoning. Clin Toxicol (Phila) 2010; 48:47-56.

28. Yang Y, Rosenberg GA. Blood-brain barrier breakdown in acute and chronic cerebrovascular disease. Stroke 2011;42:3323-8. 